\title{
Plan estratégico para el desarrollo de productos que permitan preparar a las personas en situación de calle y faciliten su reinserción sociolaboral
}

Strategic Plan for the development of products that allow the job training and facilitate the re-employment of homeless people

Paula Ortiz-Portuguez'

Fecha de recepcción: 06/04/2017

Fecha de aprobación: 20/07/2018

Paula Ortiz-Portuguez. Plan estratégico para el desarrollo de productos que permitan preparar a las personas en situación de calle y faciliten su reinserción sociolaboral. Revista IDI+ Volumen I N'I. Julio - Diciembre 2018

Pág 27-39 


\section{Resumen}

La indigencia, o mejor dicho la situación de calle, es una problemática social que se ha venido en aumento a partir del 2014 en Costa Rica. Históricamente, la atención social de esta problemática se ha caracterizado, básicamente, por ser iniciativas de personas particulares, iglesias, ONG y otros, quienes, por lo general, ofrecen ayudas que cubren únicamente las necesidades básicas (alimentación, vestimenta, etc.). Además, existen las ayudas para superar la adicción a las drogas; sin embargo, un gran número de los casos tras la superación presenta recaída. Una de las principales causas de esto es la dificultad que representa reintegrarse sociolaboralmente a la sociedad. En Costa Rica son pocos los lugares que ofrecen este tipo de preparación y ayuda; en Cartago está el Centro de Atención Integral, el cual aún no está en funcionamiento, además de que tampoco se había contemplado la posibilidad de ofrecer herramientas para la inserción laboral.

Este proyecto presenta como estrategia facilitar la formación y capacitación de las personas en situación de calle, desde un enfoque agrícola, por medio del desarrollo de productos y siguiendo una idea de diseño social. Específicamente, se trabajó con el Centro de Atención Integral de Cartago como contexto, y bajo un concepto de integración y autosustentabilidad, se propone la implementación de cuatro talleres que abarquen un periodo de aprendizaje de cuatro a seis meses.

\section{Palabras clave}

Diseño social; integración; motivación; preparación; agrícola; indigencia; situación de calle; autosustentable; diseño de producto.

\section{Abstract}

Indigence, or rather the street situation is a social problem that has been increasing from 2014 in Costa Rica. Historically social care of this problem has been characterized as initiatives from individuals, churches, ORG and others, who generally offer aid covering only basic needs (food, clothing, etc.). There are also aided to overcome drug addiction, however a large number of exceedances, presents relapse. One of the main causes of this, is the difficulty of sociooccupationally reintegration into society. In Costa Rica, there are few places that offer this type of preparation and help, but there is only one in the city of Cartago, El Centro de Atención Integral (Integral Care Center), which is not yet in operation, and neither considered the possibility of providing job readiness.

This project presents a strategy that aims to facilitate the training of people homeless, with an agricultural focus, through the development of products following an idea of social design. Specifically, with the Centro de Atención Integral of Cartago as context and under the concept of integration and self-sustainability the implementation of 4 workshops for a learning period between 4 to 6 months are proposed.

\section{Keywords}

Social design; integration; motivation; training; agricultural; homeless; self-sustaining; product design. 


\section{Introducción}

Por sus expresiones y tendencia al crecimiento, la indigencia se ha convertido en una problemática social, pues existe un marcado rechazo y discriminación hacia este segmento de la población costarricense. Datos del Sistema de Información de Población Objetivo (SIPO), del Instituto Mixto de Ayuda Social (IMAS), revelan que, para octubre del año 2014, cerca de 1092 personas vivían en estas condiciones en San José1. Y se ha venido dando un crecimiento notable de este número en tan solo un año, pues un estudio realizado por la Universidad Libre de Costa Rica (ULICORI), nos deja conocer que, para el 2015, alrededor de 1591 personas dormían en las calles de San José, estudio que solo contempla los distritos Hospital, Catedral, Merced y Carmen.

Históricamente, la atención social de esta problemática se ha caracterizado por provenir de iniciativas particulares, iglesias, ONG y otros. Por su carácter particular, con frecuencia, estas iniciativas no tienen a disposición recursos estables, lo cual hace que estos esfuerzos particulares no sean constantes, sino que dependan de las posibilidades, los recursos y la voluntad de terceros. Sin embargo, en su mayoría, estas iniciativas se enfocan en acciones destinadas a cubrir necesidades básicas: hambre, vestido, aseo personal, condicionado, en no pocas ocasiones, a recibir mensajes de naturaleza espiritual. Además de estas necesidades básicas, existen otras que no se ven a simple vista, pero que son igual de relevantes para que estas personas puedan salir de tal condición, lo mismo que las necesidades de reconocimiento y autorrealización también son de gran importancia, aunque no se cubran las necesidades más elementales.

Según Bruce M. Tharp y Stephanie M. Tharp3, hay cuatro campos o áreas del diseño que dividen nuestro quehacer: diseño comercial, diseño responsable, diseño experimental y diseño discursivo. Diseño responsable es lo que se entiende, en gran medida, como un diseño social o socialmente responsable, impulsado por una noción más humanitaria de servicio. En esta área, el objetivo del diseñador es proporcionar un producto útil, utilizable y deseable para aquellos que el mercado ignora. Cuestiones como la ética, la compasión, el altruismo y la filantropía rodean este trabajo, y es común tanto en países en desarrollo como desarrollados. Mientras que el diseño responsable puede tener -y a menudo la tiene- una relación con el mercado, "disponible comercialmente"; su intención principal no es maximizar beneficios, sino servir los mercados menos atendidos.

Al implementar este concepto de diseño social, en este artículo se expone un plan estratégico de desarrollo de productos que faciliten una efectiva reinserción sociolaboral de las personas que estarán hospedadas en el Centro de Atención Integral de Cartago, quienes son personas que han pasado por experiencias de situación de calley adicción de sustancias drogodependientes.

\section{Metodología}

\section{Recolección de datos}

Esta investigación es de tipo no experimental. Se realiza una investigación y análisis de la información existente, con el objetivo de plantear un plan estratégico que logre brindar soluciones viables a la problemática expuesta. Además, es de un enfoque cualitativo o exploratorio, porque se utilizó un 
enfoque de recolección de datos sin medición numérica, para descubrir o afinar preguntas de investigación. En ese sentido, los instrumentos utilizados son los siguientes:

1. Estudios bibliográficos: Estudios realizados con anterioridad por instituciones gubernamentales del país, encuestas, tesis de Trabajo Social o de la Escuela de Enfermería de la Universidad de Costa Rica (UCR). Además, se extrajo mucha información del Diagnóstico situacional de las personas en condición de indigencia en el cantón Central de Cartago, realizado por la Bach. Cristina Brenes Rivera y la Licda. Ana Arce Sandí, trabajadora social de la oficina de Equidad de Género y Desarrollo Social de la Municipalidad de Cartago.

2. Entrevistas coloquiales e informales: En este caso no se sigue un conjunto predeterminado de preguntas, sino que se adopta un enfoque más flexible o "sobre la marcha". Se hicieron entrevistas y reuniones en Fundación Génesis, Municipalidad de Cartago, y con el grupo Pobre de Mí, con el fin de poder valorar de qué modo abordar la problemática planteada por medio del diseño de productos.

3. Observación y seguimiento: Se llevó a cabo un acercamiento con respecto a la población en condiciones de indigencia; se repartieron alimentos con el grupo Pobre de Mí, para conocer mejor el perfil de esta población (perfil del usuario).

Para profundizar en mayor grado la investigación, se realizó un análisis de la situación actual, mediante diversos métodos que permiten determinar el potencial del proyecto, a partir de factores políticos, sociales, económicos y tecnológicos (PEST), y la capacidad instalada que tiene el país para fabricarlo posteriormente.

\section{Validación}

Luego de una recolección y procesamiento de datos, se genera la propuesta del plan estratégico, la cual es necesario validarla. Para esto se utilizaron diversos métodos de testeo y prototipado (Imagen 1).

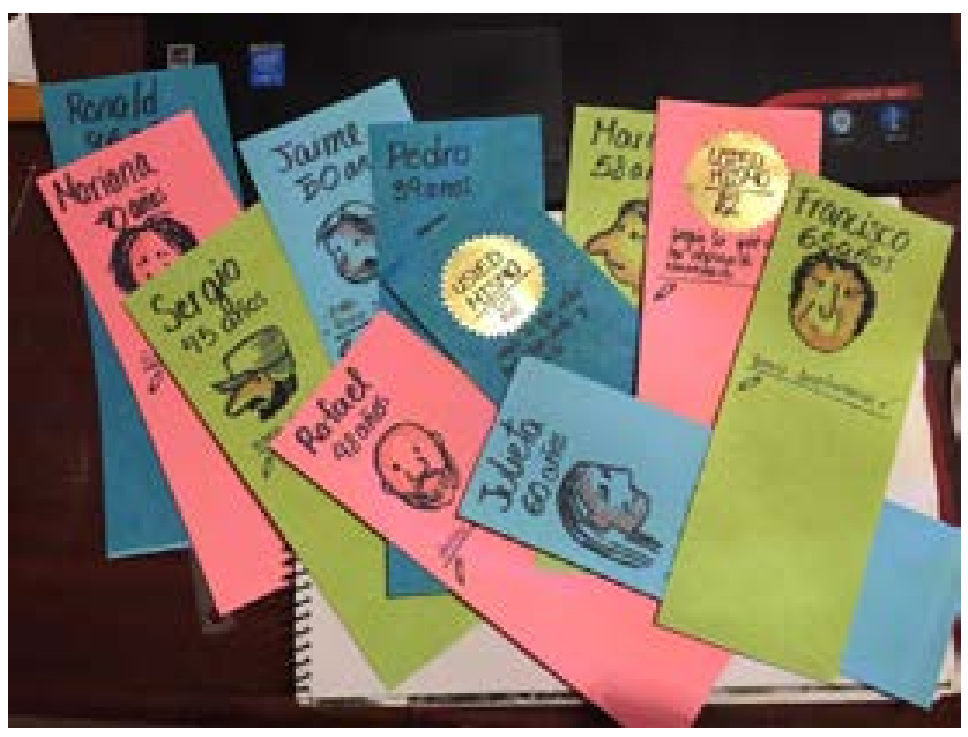

Imagen1. Personas para testeo. 
Al no encontrarse en funcionamiento el Centro de Atención Integral aún, se recurre como población para el testeo a personas que estén familiarizadas con la población meta. Personas miembros de grupos de ayuda social Pobre de Mí, del Centro Dormitorio de San José y Fundación Génesis. A pesar de no ser personas que vayan a usar el sistema, aportan información muy importante para la propuesta. Es conveniente recalcar que a la población se le pidió actuar según un cambio de roles (role playing, una forma rápida y tangible para probar una idea o experiencia, encarnando un personaje y pensando como lo haría él). Para seguir con este caso, se le dio a cada persona un papel con una PERSONA, para ayudarles a crear ese personaje. Solo se les da nombre, edad, y que pasaron por un proceso de desintoxicación, con el fin de que ellos se expresen con libertad según lo que han visto y hablado con los habitantes de la calle.

\section{Storyboard}

Se realiza un storyboard (guion gráfico), con el fin de ayudar a visualizar el concepto desde el inicio hasta el final. Este método no solo ayudará a redefinir la idea, sino que ayudará a comprender quién lo usará, cómo, dónde, etc.

\section{Prototipo de experiencia}

Este método es una simulación de la experiencia de servicio que prevé algunas de sus actuaciones mediante el uso de los puntos de contacto físicos específicos implicados. El prototipado de experiencia permite a los diseñadores mostrar y probar la solución mediante una participación activa de los usuarios.

\section{Prototipado rápido}

El prototipado rápido, cuando se trata de diseño centrado en el usuario, es un medio muy eficaz para mostrar las ideas tangibles, aprender mientras se hace, además de obtener resultados rápidos en cuanto a retroalimentación. El prototipo no es estéticamente perfecto. En relación con este proyecto, se implementaron maquetas como prototipado rápido, con el fin de demostrar el funcionamiento del sistema y la interacción de involucrados por medio de muñecos.
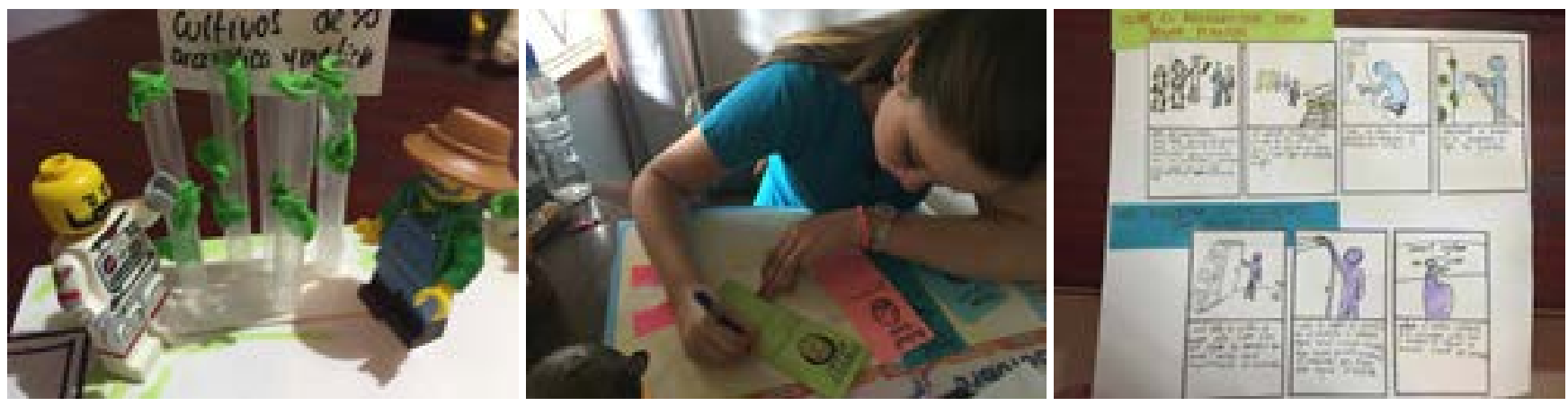

Imagen 2. Testeo con prototipado rápido, uso de PERSONAS y storyboard.

\section{Protocolos de pensamiento en voz alta:}

Se aplicó como complemento del rapid prototyping y experience prototyping, un valioso método para identificar las preocupaciones, deseos y motivaciones de los usuarios de determinados productos, servicios y sistemas. 


\section{Resultados}

Se ordenaron los datos recopilados y se identificaron patrones y similitudes útiles para la etapa de conceptualización. Posteriormente se da una interpretación por medio de gráficos, cuadros, infográficos y tablas, con el objetivo de obtener un panorama general de la situación actual de los involucrados y tendencias. Luego se definen tres ejes, los cuales son base de todo el proyecto y se pueden ver en la Imagen 3.

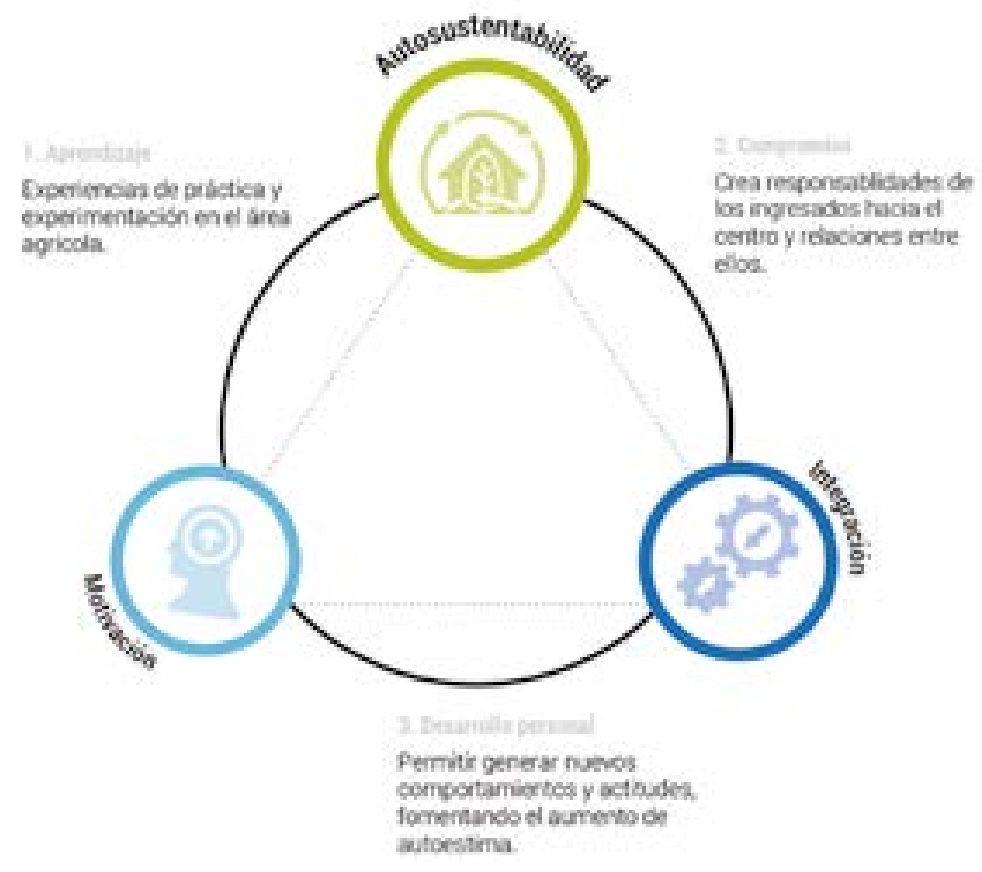

Imagen 3. Ejes del proyecto.

Finalmente se definieron los talleres por implementar y las etapas para que esta estrategia se puede llevar a cabo:

\section{ETAPA 1}

La finalidad de esta etapa es comunicar a la Municipalidad de Cartago la importancia y el impacto positivo que este proyecto traerá al Centro de Atención Integral. Una vez aceptada la propuesta, se planifica la gestión del proyecto (se inicia la búsqueda de recursos financieros para armar un presupuesto, investigar, definir alianzas para el desarrollo de ser necesario y generar los productos en detalle.) 


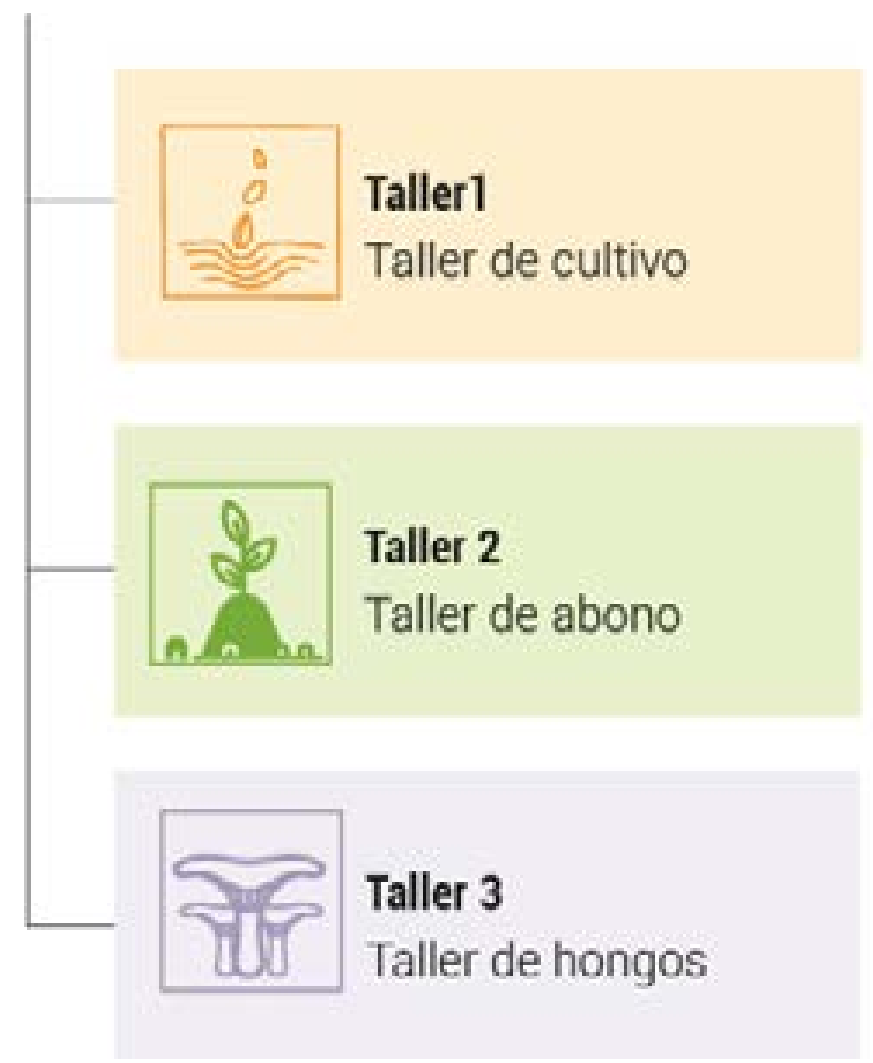

Imagen 4. Etapa 1.

\section{ETAPA 2}

El propósito de esta etapa es realizar los talleres de capacitaciones. Esta etapa es en la que se desarrollarán los productos propuestos, con el fin de que faciliten la enseñanza y aprendizaje de capacitaciones agrícolas en el Centro de Atención Integral. En esta etapa se procura establecer la contratación con empresas que fabriquen los productos que se plantearon.

\section{ETAPA 3}

Esta etapa tiene como objetivo es que los ingresados al Centro puedan ofrecer los productos que se generen en los talleres en las Ferias del Agricultor, ferias orgánicas o de emprendedores. Por tanto, en esta etapa se requiere promover alianzas y obtener los permisos necesarios para la venta en una de las ferias mencionadas. Además, es importante el desarrollo de una marca y el empaque.

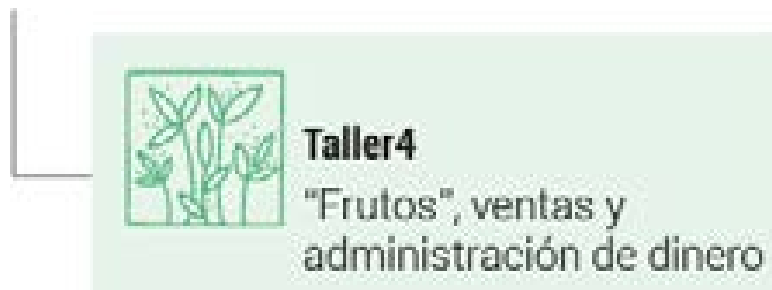

Imagen 5. Etapa 3.

Asimismo, para cada taller de capacitación mencionado anteriormente, se desarrollarán los siguientes productos: 


\section{Producto 1. Sembradío vertical}

Importancia del producto

La importancia de este producto es meramente emocional. Es importante que los ingresados sientan conexión o sentido de pertenencia con este producto y se sientan orgullosos de lo que hacen ahí y quieran enseñarlo a los visitantes.

Según los objetivos del Centro planteados, su importancia también recae en facilitar a otras organizaciones estatales u ONG dar asistencia a estas personas en términos de orientación, terapias, cursos libres, y cualquier otra forma de apoyo que pueda coadyuvar en el abandono de las situaciones de indigencia y/o adicción.

\section{Resultados esperados del Taller 1}

Crear una sensación de pertenencia, responsabilidad.

Aprender a cultivar plantas aromáticas y medicinales con dos métodos.

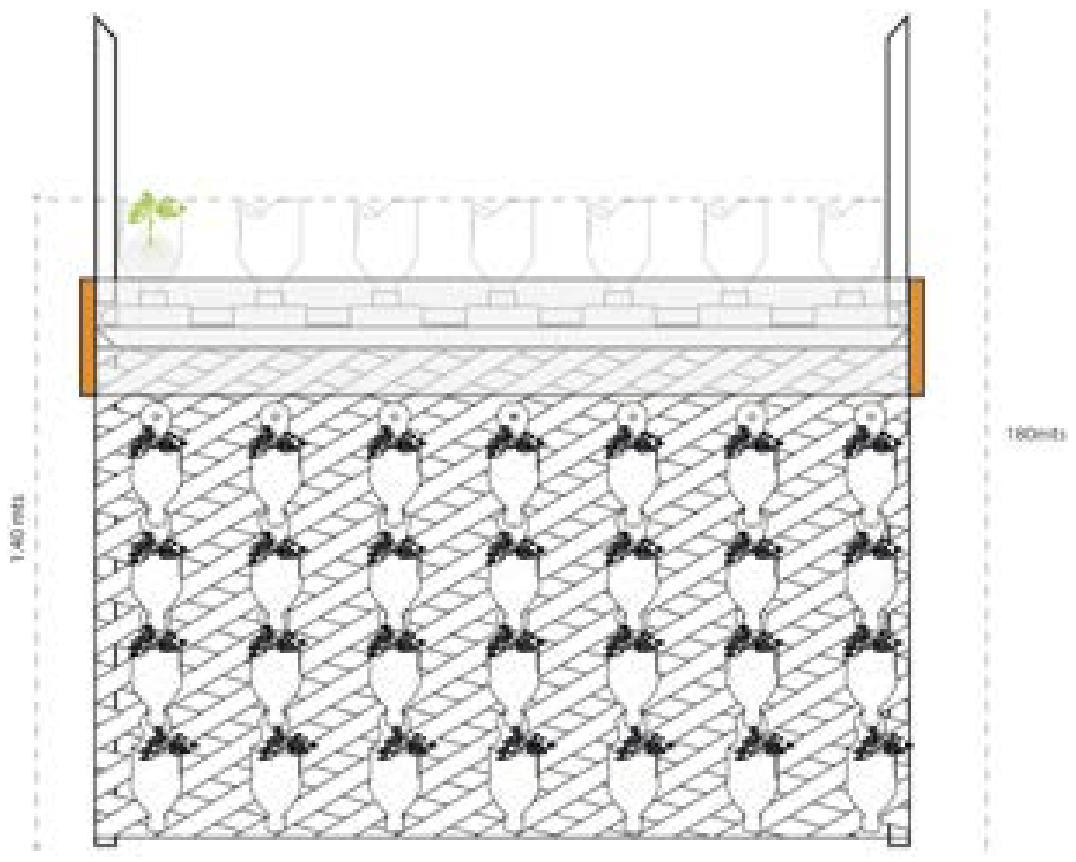

Imagen 6. Sembradío vertical.

\section{Producto 2. Recolector de agua pluvial Importancia del producto}

Recolectar el agua de lluvia para un sistema autosustentable, pues lo ideal es contar con los elementos necesarios para el riego de los cultivos en los talleres.

Integración de los demás talleres.

\section{Producto 3. Compostero}

Importancia del producto

Con respecto a este producto, es importante que se logre relacionar el bien que hace el abono a su planta, del mismo modo que ellos reciben ayuda del Centro que los ayudará a tener una mejor calidad de vida.

Aprenden el método para producir abono orgánico y así logran adquirir otro conocimiento que puedan desarrollar después. 


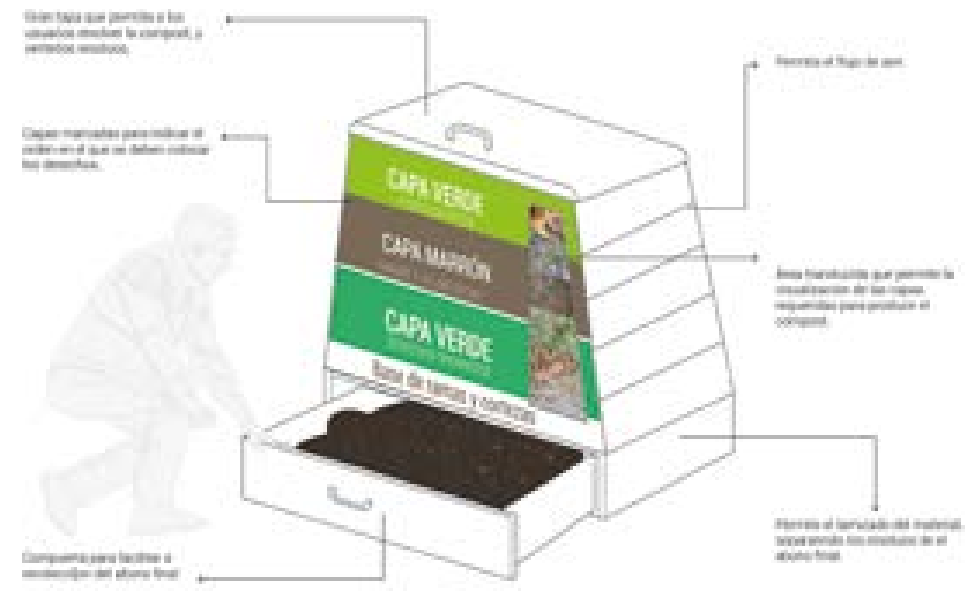

Imagen 7. Compostero.

\section{Producto 4. Incubadora de hongos ostra \\ Importancia del producto}

En este taller se trabajaría en conjunto con una analogía de integración social, por lo que es importante generar esta relación, con el fin de propiciar el trabajo en equipo.

Según los objetivos que se ha planteado el Centro, su importancia también recae en facilitar a otras organizaciones estatales u ONG dar asistencia a estas personas, en aspectos como orientación, terapias, cursos libres, y cualquier otra que pueda contribuir al abandono de las situaciones de indigencia $y / o$ adicción.
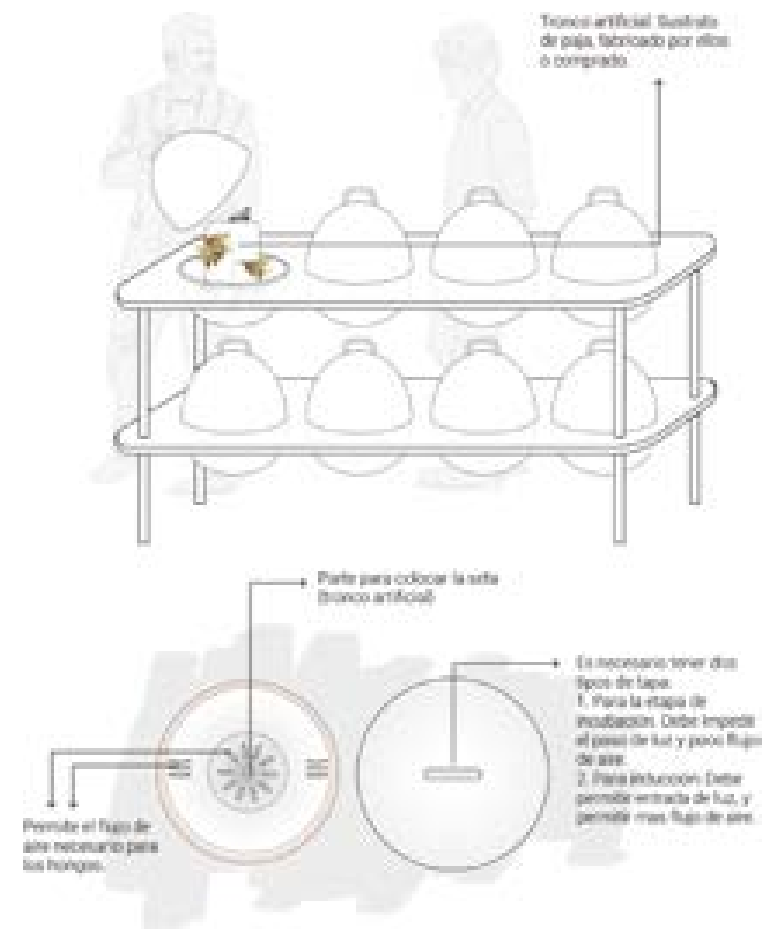

Imagen 8. Incubador de hongos ostra. 


\section{Producto V. Carné y sistema de adhesivos.}

Importancia del producto

- Generar un apoyo visual a un plan de recuperación que tiene como objetivo lograr superar la adicción, siguiendo los 12 pasos con los que trabajan Alcohólicos Anónimos, o cualquier otro paso que resulte conveniente.

- Reconocer como importante el logro que hacen los ingresados, por medio del sistema de recompensas (reward system); por esto se planea que cada vez que completen un taller, se les dé un adhesivo que represente la finalización de ese taller.

- Incorporar de la parte espiritual.

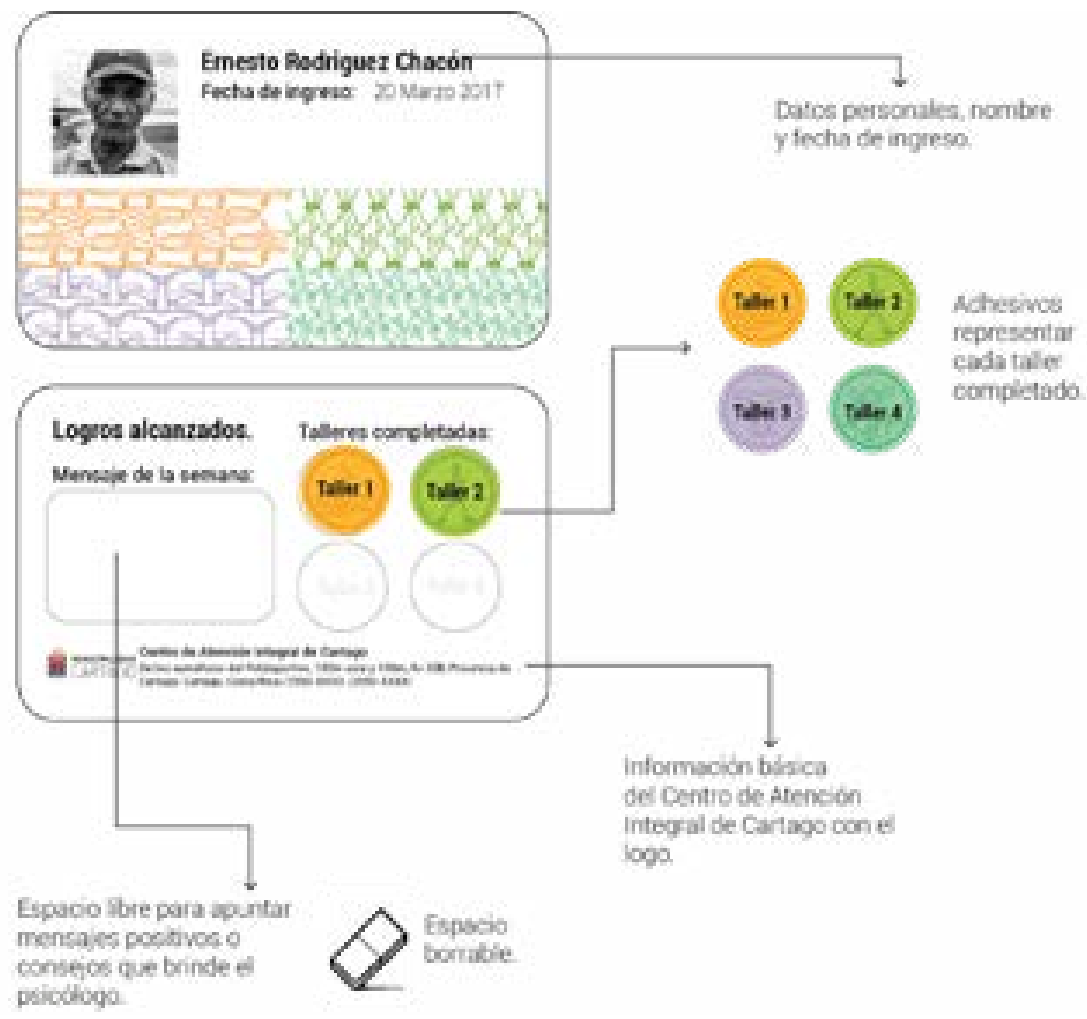

Imagen 9. Carné.

\section{Conclusiones}

1. Ha sido muy interesante el desarrollo de este proyecto, en especial porque todas las personas a quienes se entrevistó en la etapa de investigación y prototipado no sabían cómo podría contribuir el diseño de productos a una problemática social de esta magnitud. El proyecto posee una relación con la parte de ayuda social, y se presenta como una opción posible de implementar en el Centro de Atención Integral de Cartago.

2. Considerando que deben desarrollarse con base en el concepto de autosustentabilidad, resulta propicio conocer que en el país se ofrecen materiales plásticos que hechos a base de plástico reciclado. 
3. La parte de testeo y prototipado generó dificultades, principalmente porque, si se quiere entrevistar a esa población, la gran mayoría no va a querer colaborar. Esto se vio reflejado en el diagnóstico que realizó la Municipalidad en el 2013. En este caso, lo que se hizo fue acompañar al grupo Pobre de Mí a repartir comida entre la población indigente, en Cartago centro, y, aprovechando la investigación etnográfica, se conversó con algunos y se observó lo que hacían y cómo. Además, se solicitó ayuda a personas que han estado en mucho contacto con estas personas, como trabajadores sociales, agrupaciones de ayuda social, psicólogos y voluntarios. Ellos dieron información con respecto a lo que han escuchado y observado de ellos.

4. El diseñador industrial juega un papel fundamental en el desarrollo del plan estratégico, desdeel diseño de productos, hasta la implementación de la estrategia, lo que permite una integración entre los productos y los usuarios con el Centro.

5. Establecer relaciones, alianzas o vínculos con el Instituto Nacional de Aprendizaje (INA) se considera primordial.

6. Los productos aquí propuestos están enfocados a satisfacer las necesidades del Centro y las de esta población. No obstante, con sus respectivos ajustes tienen un potencial de comercialización.

7. Los cursos que se pensaron son excelentes para el contexto porque no requieren equipo ni mucho material; además, se producen recursos que pueden ser aprovechados por el Centro o vendidos.

\section{Recomendaciones}

1. La principal recomendación que se le hace al Centro es que, luego de la realización del plan piloto, efectuar pruebas y ajustar la estrategia con los cambios que se crean pertinentes con respecto a las etapas 2 y 3. Por lo tanto, se aconseja hacer un seguimiento en cada taller por un periodo de dos meses, con el fin de evaluar que, efectivamente, se alcancen los resultados esperados, y en caso de ser necesario, realizar las mejoras pertinentes en el mes siguiente.

2. Se recomienda, en algún momento, el desarrollo de mobiliarios de trabajo abatibles, que permitan el almacenaje de los materiales y herramientas, y a la vez, provean una zona de trabajo. Esto, con el fin de disponer de todos los objetos necesarios para las actividades que se van a llevar a cabo en cada taller.

3. Para el taller 3, es pertinente realizarlo en un espacio con techo (tensoestructura), con espacio para sentarse (véase la siguiente imagen). Incluso se puede contemplar realizar dos: uno para el taller 3 y otro únicamente para charlas, o impartir la parte teórica de los demás talleres. Tiene que dar una sensación de parada (stop), un lugar de recreación para que así llame la atención y motive a ir, con lo cual se elimina la noción del típico taller de cuatro paredes, caluroso e incómodo.

4. Además, es importante considerar la posibilidad de hacer rotulaciones que contengan instrucciones de uso; es decir, qué se puede hacer y qué no en cada taller. 
5. Con respecto al taller 4 , la idea es que ellos retomen un hábito de trabajo y que aprendan a administrar su dinero. Por tanto, se recomienda que los usuarios tengan asesoramiento por parte de algún empleado bancario, con el fin de generar un fondo de ahorro, el cual ya estará disponible para la siguiente etapa del plan piloto, que es la búsqueda de empleo.

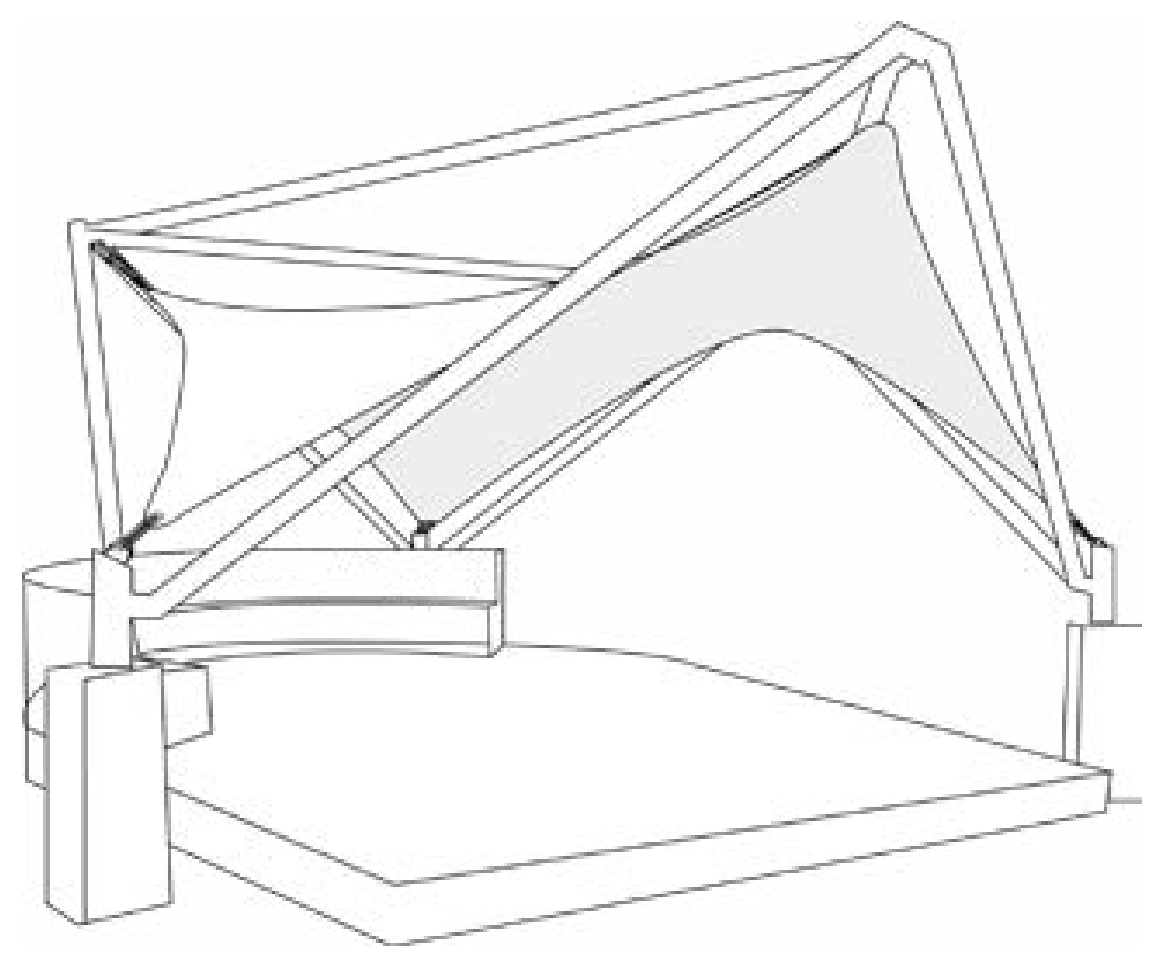
Imagen 11. Tensoestructura.

\section{Bibliografía}

Chinchilla C., Sofía. "Indigencia atrapa a más mujeres y jóvenes en la calle". La Nación 27, Marzo del 2016: 4A, 5A. Print.

Comisión Intersectorial contra el Abuso Sexual y la Explotación Sexual Comercial de Niños, Niñas y Adolescentes (CICAES) (2006). Caracas. Recuperado de: http://www.annaobserva.org/ckfinder/userfiles/ files/POLITICAS\%20P\%C3\%9ABLICAS\%20VENEZUELA. Pdf.

García Allen, J. (s. f.). Pirámide de Maslow: La jerarquía de las necesidades humanas. Recuperado de: https://psicologiaymente.net/psicologia/ piramide-de-maslow\#! Granados

Hernández, M. y Ramírez-Campos, J. (2013). “Condiciones y experiencias de vida de las mujeres gestantes indigentes que viven en el casco noroeste metropolitano de San José". Revista Enfermería Actual de Costa Rica, 25, 1-16. Recuperado de: <http://www.revenf.ucr.ac.cr/ indigencia. Pdf.

Leitón, P. (2016). “Desempleo alcanzó 9,5\% en el primer trimestre de este año". nacion.com. Recuperado el 31de julio de 2016 de: http:// www.nacion.com/economia/indicadores/empleo-inecdesempleo_ 0_1560244021.html 
Masqueunacasa. (2016). Espacios multifuncionales. Masqueunacasa.org. Recuperado el 10 junio 2016, de: http://masqueunacasa.org/es/ habitapedia/propuestas/espacios-multifuncionales. Pdf.

M. Tharp, B. and M. Tharp, S. (2018). The 4 Fields of Industrial Design: (No, not furniture, trans, consumer electronics, \& toys), by Bruce $M$. Tharp and Stephanie M. Tharp - Core77. [online] Core77. Available 11- 6- 2016 at: http://www.core77.com/posts/12232/the-4-fieldsof-industrial-design-no-not-furniture-trans-consumer-electronicstoys-by-bruce-m-tharp-and-stephanie-m-tharp-12232. Pdf.

Organización Internacional del Trabajo (OIT) (s. f.). ERGONOMÍA. Training. itcilo.it. Recuperado el 10 de junio de 2016, de: http://training.itcilo.it/ actrav_cdrom2/es/osh/ergo/ergonomi.htm. Pdf.

Ruiz, P. (2016).“Muebles para Espacios Pequeños: Grandes en Optimización, Versatilidad y Confort". Revista M\&M, 66-74. Recuperado el 10 junio 2016, de: http://www.revista-mm.com. Pdf.

Seldon, K. (2008). Design lesson: Small space strategies. Style at Home. Recuperado el 10 junio 2016, de: http://www.styleathome.com/ decorating-and-design/design-lesson/design-lesson-smallspacestrategies/a/546

Solano, C., \& Arce, A. (s. f.). Programa de atención de personas en condición de indigencia del cantón Central de Cartago. Material no publicado.

Téllez Rojas, E. (2006). “El diseño y la multifunción de los objetos”. Catálogo Digital de Publicaciones D. C. Fido.palermo.edu. Recuperado el 10 junio 2016, de: http://fido.palermo.edu/servicios_dyc/ publicacionesdc/vista/detalle_articulo.php?id_articulo=5208\&id_ libro=13. Pdf.

Torres, Jason. (2016).“Indigencia prolifera sin control en casco metropolitano de San José. CRHoy.com, periódico digital. Costa Rica Noticias, 24/7, 30 de marzo de 2016.

Ugarte, Joselyne. (2016) "IAFA preocupado por aumento de adolescentes en indigencia". Crhoy.com, periódico digital. Costa Rica, Noticias 24/7, 30 marzo de 2016.

Vega, A. (2014). "10 secretos para decorar espacios pequeños". El Blog del Decorador. Recuperado el 10 junio 2016, de: http://www. elblogdeldecorador.cl/2014/08/10-secretos-para-decorar-espaciospequenos. Pdf.

Young, Dominick (2014).“Indigencia en Costa Rica”. Batmanandrobincc1701. blogspot.com. N.p. Recuperado el 30 marzo de 2016, de: http:// batmanandrobincc1701.blogspot.com/2014/05/la-indigenciapara-el-estado-y-otras.html. 